\title{
Superemission in vertically-aligned single-wall carbon nanotubes
}

\author{
Igor Khmelinskii ${ }^{\text {a }}$, Vladimir Makarov ${ }^{\mathrm{b}, *}$ \\ a University of the Algarve, FCT, DQB and CIQA, 8005-139 Faro, Portugal \\ b University of Puerto Rico, Rio Piedras Campus, PO Box 23343, San Juan, PR 00931-3343, USA
}

Received 4 April 2016; received in revised form 17 July 2016; accepted 18 July 2016

Available online 25 July 2016

\begin{abstract}
Presently we used two samples of vertically aligned single-wall carbon nanotubes (VA SWCNTs) with parallelepiped geometry, sized $0.02 \mathrm{~cm} \times 0.2 \mathrm{~cm} \times 1.0 \mathrm{~cm}$ and $0.2 \mathrm{~cm} \times 0.2 \mathrm{~cm} \times 1.0 \mathrm{~cm}$. We report absorption and emission properties of the VA SWCNTs, including strong anisotropy in both their absorption and emission spectra. We found that the emission spectra extend from the middle-IR range to the near-IR range, with such extended spectra being reported for the first time. Pumping the VA SWCNTs in the direction normal to their axis, superemission (SE) was observed in the direction along their axis. The SE band maximum is located at $7206 \pm 0.4 \mathrm{~cm}^{-1}$. The energy and the power density of the superemission were estimated, along with the diffraction-limited divergence. At the pumping energy of $3 \mathrm{~mJ} / \mathrm{pulse}$, the SE energy measured by the detector was $0.74 \mathrm{~mJ} / \mathrm{pulse}$, corresponding to the total SE energy of $1.48 \mathrm{~mJ} /$ pulse, with the energy density of $18.5 \mathrm{~mJ} \mathrm{~cm}^{-2} /$ pulse and the SE power density of $1.2 \times 10^{5} \mathrm{~W} \mathrm{~cm} / \mathrm{cm}^{-2} / \mathrm{pulse}$. We report that a bundle of VA SWCNTs is an emitter with a relatively small divergence, not exceeding $3.9 \times 10^{-3} \mathrm{rad}$. We developed a theoretical approach to explain such absorption and emission spectra. The developed theory is based on the earlier proposed SSH theory, which we extended to include the exchange interactions between the closest SWCNT neighbors. The developed theoretical ideas were implemented in a homemade FORTRAN code. This code was successfully used to calculate and reproduce the experimental spectra and to determine the SWCNT species that originate the respective absorption bands, with acceptable agreement between theory and experiment.
\end{abstract}

Published by Elsevier B.V.

Keywords: A Nanostructures; B Composites; C Optical properties; D Electron microscopy; D Electronic structure

\section{Introduction}

Many earlier studies were devoted to the unique optical properties of carbon nanotubes [1-3]. Single-walled carbon nanotubes (SWCNTs) are a special and very interesting material, as regards their optical properties. Their diameter is typically in the $1.0-5.0 \mathrm{~nm}$ range, with

\footnotetext{
* Corresponding author. Tel.: +1 787529 2010; fax: +1 7877647717 .

E-mail address: vmvimakarov@gmail.com (V. Makarov).
}

the tube length significantly exceeding its diameter. The structure of a SWCNT may be characterized by a pair of integer numbers $(n, m)$, which define the vector of the system translational symmetry:

$\vec{r}=m \vec{a}_{1}+n \vec{a}_{2}$

where $\boldsymbol{a}_{1}$ and $\boldsymbol{a}_{2}$ are the primitive vectors of the graphene lattice cell. The nanotubes have a zigzag structure when 
$m=0$, and an armchair structure when $n=m$. The diameter of an ideal nanotube may be calculated as follows:

$d=\frac{a}{\pi} \sqrt{(m+n)^{2}-m n}$

where $a=0.246 \mathrm{~nm}$.

The SWCNT properties change significantly in function of the $(n, m)$ values, in a non-monotonous manner [3]. For example, the band gap in the SWCNTs varies from zero to about $2 \mathrm{eV}$, with their electric conductivity showing either metallic or semiconducting behavior. These fundamental properties very strongly affect their optical properties.

The density of states (DOS) in semiconducting carbon nanotubes is independent of the translational unit cell size and the chiral angle [3-6]. The absorption spectra and the electron energy loss spectra (EELS) of SWCNT bundles were calculated, with the energies of the three main peaks given approximately by [3-6]:

$E_{l}=\frac{2 l a}{d}\left|V_{p p \pi}\right|, \quad l=1,2,3$

where $V_{p р л}$ is the $p p \pi$ transfer integral between the two nearest carbon atoms. The $p$ orbital rehybridization effect on the peak position was extensively studied as well [7-10]. It was found that such rehybridization may significantly change the DOS of the SWCNTs. Chen et al. [11] took these effects into account in their calculations, obtaining a good agreement between theoretical and experimental spectra. A theoretical analysis of the detailed structure of the SWCNT absorption bands was carried by Prylutsky et al. [12]. They found that specific groups of subbands appear in different parts of the SWCNT absorption spectrum, with the resulting spectrum significantly more complex than the spectra than only have three principal absorption bands. Therefore, it is very important to discuss the experimental measurements of the SWCNT absorption spectra. Meyyappan [13] described the absorption spectra of the high purity metallic and semiconducting SWCNTs, separated using the density-gradient ultracentrifugation. The spectra were recorded in the wide energy range from $1 \mathrm{meV}$ to $5 \mathrm{eV}$. A strong and broad absorption band was observed at $0.06 \mathrm{eV}$ in a high-purity metallic nanotube sample. This observation suggests that the optical properties of even the high-purity metallic nanotube bundles cannot be described by the simple Drude conduction model. The origin of these absorption bands for the metallic and semiconducting nanotube samples was discussed [14], taking into account the small energy gap in the metallic nanotube bundles and plasmonic resonance. Leson et al. [15] carried out an experimental investigation of the optical spectra of the SWCNTs at temperatures up to $1273 \mathrm{~K}$. Their studies give insights into the electronic structure of the metallic and semiconducting SWCNTs at different temperatures by measuring the shifts of the $S_{11}, S_{22}$ and $M_{11}$ bands using optical absorption spectroscopy, where $\mathrm{S}$ refers to the semiconducting SWCNTs and M to the metallic SWCNTs.

A decrease in the transition energies in both metallic and semiconducting SWCNTs with temperature is determined by the temperature shift of the $S_{11}, S_{22}$ and $\mathrm{M}_{11}$ absorption bands. Weisman et al. [16] analyzed the absorption spectra of the SWCNTs scanned over a wide spectral range. The broad backgrounds in the visible-near-IR absorption spectra were studied in a series of tightly controlled experiments [16], reporting no major differences between the spectral backgrounds in the sample fractions with the average lengths between 120 and $650 \mathrm{~nm}$. Thus, the authors concluded that the broad background absorption is created by an amorphous carbon fraction present in the SWCNT samples [16]. Takeyama et al. [17] performed measurements of the optical absorption cross-section of the SWCNTs. They provided an empirical formula allowing to predict the absorption spectrum of any SWCNT. A detailed analysis of the above reports shows that the SWCNTs demonstrate very interesting optical properties, which justify an additional research effort. Lately we demonstrated [18] that metal nanolayers produce superemission; we therefore expected to obtain similar effects in SWCNTs as well.

Single-walled carbon nanotubes are a very promising material for broad technological applications [19,20]. The diameters of SWCNTs vary in the range of $1-5 \mathrm{~nm}$ and the length exceeds the diameter by the factor of $10^{2}-10^{7}$. Ugawa et al. [21] studied the MIR energy gap in the SWCNTs. They found that the energy gap of metal-like SWCNTs is about several thousand $\mathrm{cm}^{-1}$. Thus, the lowest-energy transitions in the absorption and emission spectra should be located in the MIR spectral region. The electronic structure of the SWCNTs was also studied quite extensively using optical spectroscopy and $a b$ initio calculations [22-24]. The perturbations in the common conjugated $\pi$-system were found to increase dramatically for thinner SWCNTs. The optical absorption and emission, and the excited state dynamics of SWCNTs were also investigated in detail [25-47], with the emission spectrum of the individual SWCNTs typically appearing in the NIR range, and the dynamics developing in the subpicosecond time range. However, the radiative lifetime of the relaxed system is typically in the nanosecond time range. 
The optical properties of different SWCNT isomers were also studied [48], with the differences in optical properties of different isomers of single-chirality SWCNTs used to separate these isomers. A method to determine the SWCNT diameter was also proposed and used to characterize the SWCNT morphology [49].

Presently, we report experimental and theoretically calculated absorption and emission spectra of the vertically aligned (VA) semiconductor SWCNTs $(m \neq 0$, $n \neq 0$ ). Both absorption and emission spectra of the VA SWCNTs demonstrate very strong anisotropy as regards the locations of the peak maxima and the peak widths. We also found that VA SWCNTs produce strong superemission in the direction of the tube axis. We also report that in the macroscopic bundle of the VA SWCNTs the emission spectra appear in the near-IR spectral range, in agreement with the NIR-only emission obtained for individual SWCNTs. We describe the observed absorption spectra considering the cooperative effects in this macroscopic system. Thus, we report the absorption, emission and superemission spectra of the VA SWCNTs, and propose theoretical models explaining the experimental results.

\section{Experimental methods}

\subsection{Samples}

The experimental measurements of the absorption, emission and superemission spectra were performed for samples consisting of bundles of vertically-aligned SWCNTs with $0.02 \mathrm{~cm} \times 0.2 \mathrm{~cm} \times 1.0 \mathrm{~cm}$ size (sample $\mathrm{T} 1$ ) and $0.2 \mathrm{~cm} \times 0.2 \mathrm{~cm} \times 1.0 \mathrm{~cm}$ size (sample T2) from Firstnano (www.firstnano.com), with the nanotube length of $1.0 \mathrm{~cm}$, grown in the central zone of the fused silica substrates $(1.25 \mathrm{~cm} \times 1.25 \mathrm{~cm} \times 0.05 \mathrm{~cm})$. The average SWCNT diameter was $3.7 \mathrm{~nm}$, and their number density ca. $1.7 \times 10^{5} \mu \mathrm{m}^{-2}$. The upper ends of the SWCNTs were trimmed to the same length using $600 \mathrm{eV}$ Cs ion beam, with the upper surface of the bundle parallel to the substrate. Thus, the lengths of all of the SWCNTs were essentially equal. The substrates with the SWCNT bundles were mounted on a support, with a second fused silica plate protecting the upper ends of the SWCNTs from damage, mounted with a spacing of several $\mu \mathrm{m}$ (see Fig. 1). The spectra of the samples could be recorded using different geometries. The emission was always captured in the direction normal to the excitation beam.

To characterize the samples, we recorded the SEM image of the T1 sample (JSM-7800F Extremeresolution Analytical Field Emission SEM, Jeol Inc.).

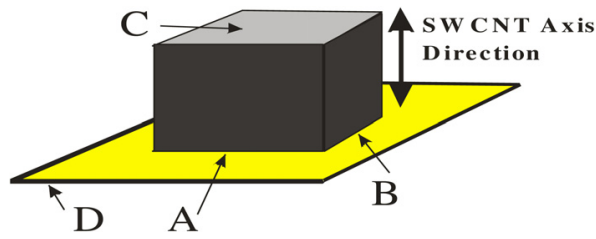

Fig. 1. The sample geometry: (A, B) the lateral faces of the vertically aligned SWCNT bundle; (C) the upper face of the vertically aligned SWCNT bundle; (D) the fused silica substrate.

The $0.2 \mathrm{~cm} \times 1.0 \mathrm{~cm}$ sample face was scanned, with the SEM image shown in Fig. 2.

The SEM image shows well-aligned SWCNTs, with the average diameter as estimated from the image being $4.3 \pm 1.8 \mathrm{~nm}$, in acceptable agreement with the producer specifications.

\subsection{The experimental methods and techniques}

The absorption and emission spectra were recorded on a Hitachi U-3900H UV-Visible and Shimadzu UV3600 Plus Spectrophotometers, and FTIR spectrometer (IFS 66/S, Bruker Inc., with LN2 cooled detectors), respectively. The spectral peak maxima and widths were calculated using the PeakFit software (Sigmaplot). The samples were reproducibly installed into a support of blackened aluminum.

Fig. 3 shows the block diagram of the experimental setup used for measurements of the superemission spectra. A high-pressure $100 \mathrm{~W}$ mercury lamp was used as the light source (ESI 1200 100W MSR Lamp 3.8 A; Planet Bulb Inc.), the radiation was focused by appropriate lenses, filtered by a water-cooled glass filter to

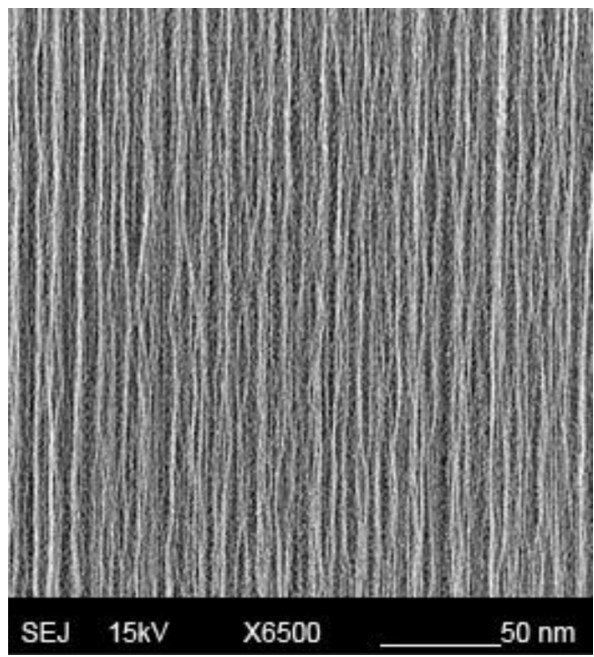

Fig. 2. The SEM image of the T1 sample, showing a bundle of vertically-aligned single-walled carbon nanotubes. 


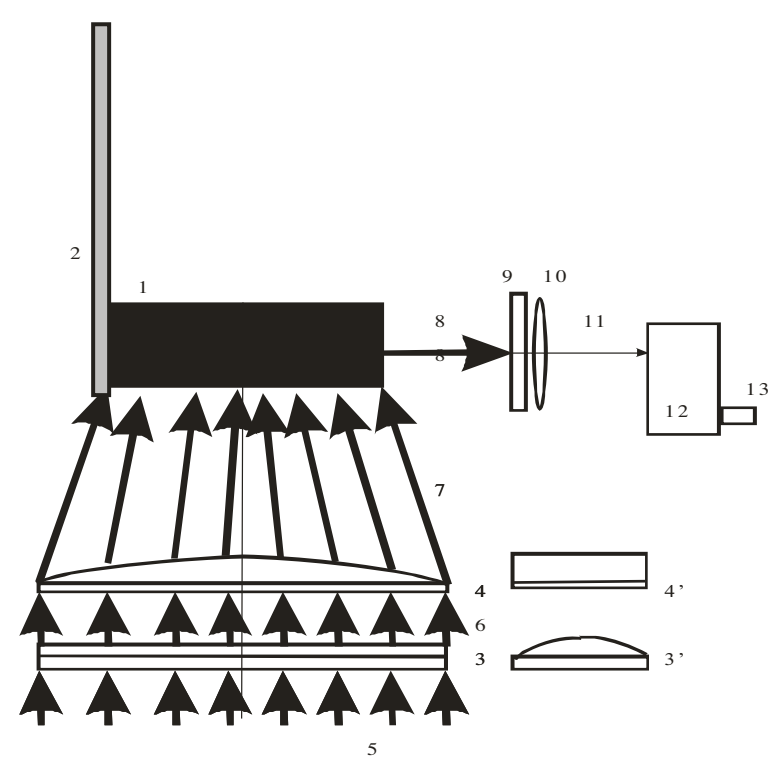

Fig. 3. Superemission measurements: 1, the face of the SWCNT sample; 2, the substrate; $3,10 \mathrm{~cm}$ focal distance cylindrical lens (viewed from the top); $3^{\prime}$, the same, viewed from the left side; $4,30 \mathrm{~cm}$ cylindrical lens (viewed from the top); $4^{\prime}$, the same, viewed from the left side; $5,6,7$, the excitation laser beam focused on the sample face; 8 , superemission exiting the top face of the sample; 9 , condenser lens; 10 , spherical lens ( $5 \mathrm{~mm}$ or $30 \mathrm{~cm}$ focal distance); 11 , sample emission passing through 9 and 10;12, monocromator; 13, PD1 detector.

separate the $313 \mathrm{~nm} \mathrm{Hg}$ emission band, and focused into a $0.2 \mathrm{~cm} \times 0.1 \mathrm{~cm}$ spot at the sample location, using an appropriate slit. The IR-NIR superemission was collected by a $\mathrm{CaF}_{2}$ lens onto the entrance slit of the monochromator (Cornerstone 130 1/8m Monochromators, NewPort Inc.), and detected by a photodiode (PD1; DET10A Si biased detector, THORLABS). The output of the PD1 signal was digitized by PCI-6034E DAQ I/O board (National Instruments), with the control code in the LABVIEW environment running on a Dell PC. The NIR and middle IR emission was focused onto the input slit of the FTIR spectrometer, the spectra were scanned and the data collected by a PC computer.

For superemission measurements, an additional experimental setup was used. An additional excitation source used a frequency-doubled (BBO crystal) dye laser (LPD-2000, $\Lambda$-Physics, with Pyrromethene-556 dye, $540-580 \mathrm{~nm}$ range; frequency-doubled radiation at 270-290 nm) pumped by a XeCl excimer laser (LPX200, $\Lambda$-Physics).The laser pulse was about $15-20 \mathrm{~ns}$ long. The dye laser radiation was focused by a cylindrical lens onto either A or B face of the sample (Fig. 1). The superemission was collected by a $30-\mathrm{cm}$ spherical $\mathrm{CaF}_{2}$ lens and registered by a photodiode (PD1; DET10A Biased Si Detector from THORLABS), after passing through a neutral density filter and a monochromator (Cornerstone 130 1/8 m Monochromator, NewPort Inc.). The data acquisition system was controlled by a PC computer, using a digital oscilloscope (WaveSurfer 400 from LeCroy), digital delay generators (DG-535, Stanford Research), boxcar integrators (SR-250, Stanford Research), a fast amplifier (SR-240, Stanford Research), and a computer interface board (SR-245, Stanford Research). The superemission spectra were scanned at $1 \mathrm{~cm}^{-1}$ resolution. The superemission energy was measured using a calibrated photodiode (DET10A Si biased detector, THORLABS). The presently developed experimental methods allowed recording the time evolution and the spectra of the superemission with high accuracy, $2.5 \mathrm{~ns}$ and $1.5 \mathrm{~cm}^{-1}$, respectively.

\section{Experimental results}

\subsection{Absorption spectra}

The absorption spectra were recorded, shown in Figs. 4-6.

Both T1 and T2 samples (Figs. 4 and 5) produced spectra composed of narrow discrete bands, with the average bandwidth of $112 \pm 10 \mathrm{~cm}^{-1}$, and the band maxima positions independent on the sample optical length. Additionally, two broad bands $3670 \pm 130 \mathrm{~cm}^{-1}$ wide are visible in the thinner $\mathrm{T} 1 \mathrm{sample}$. The relative intensities of the narrow bands are quite different in the two samples. The band maxima and intensities (in the O.D. units) are listed in Table 1, calculated using the Peak-Fit package. Taking into account that the SWCNT density is $1.7 \times 10^{13} \mathrm{~cm}^{-3}$, their absorption cross section was estimated using the Lambert-Beer law:

$D=\sigma n l$,

with $D$ the optical density, $\sigma$ the absorption cross-section, $n$ the density of SWCNTs, and $l$ the optical path length. The estimated values of the absorption cross-sections are also listed in Table 1 . The ratio of the absorption cross sections of the two samples is additionally shown in the last column. The intensity ratio that most differs from unity is the one obtained for the $22896 \mathrm{~cm}^{-1}$ band (number 3 in Table 1). The narrow spectral bands may be assigned to different $(m, n)$ configurations of the semiconducting SWCNTs, with the electronic transitions induced between discrete electronic states, while the continuum spectrum may be attributed to the metallic SWCNTs. Later on we shall simulate the absorption spectra of different types of SWCNTs.

The absorption spectrum of the T2 sample measured along the VA SWCNT axis direction is shown in Fig. 6. 

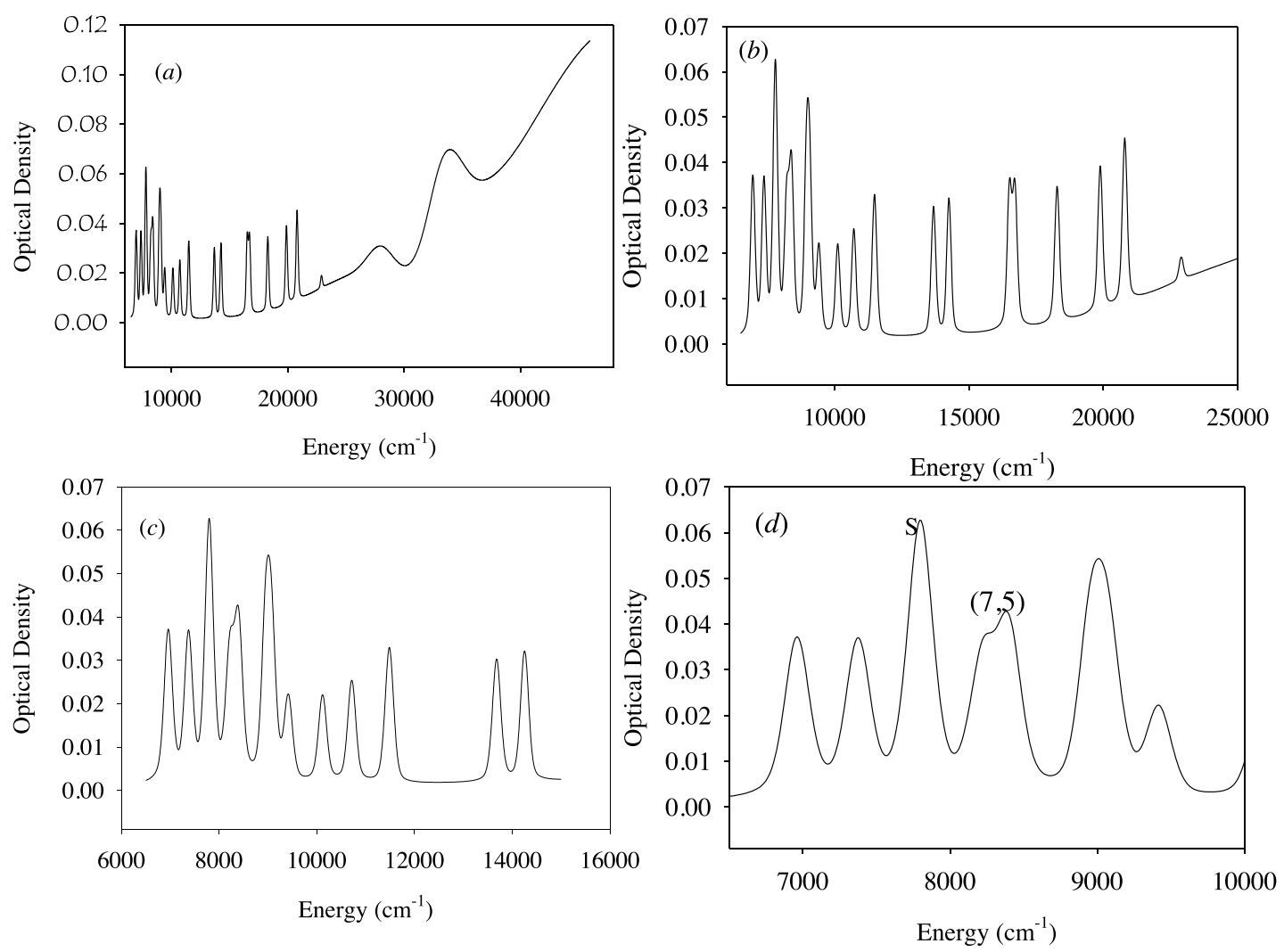

Fig. 4. The absorption spectra of the T1 sample, presented in different spectral ranges. The spectra were obtained with the probe beam normal to the SWCNT axis; the spectrum was recorded through the $0.2 \mathrm{~cm} \times 1.0 \mathrm{~cm}$ sample faces with the $0.02 \mathrm{~cm}$ optical thickness.

This spectrum is completely different from those of Figs. 4 and 5. It has a broad transmission band in the visible and near UV, with a higher absorbance outside of this window (curve 1 in Fig. 6). This transmission band was represented by a superposition of two Gaussian subbands with the following maxima, widths and amplitudes: $\omega_{1}=25341 \mathrm{~cm}^{-1}, \quad \gamma_{1}=5612 \mathrm{~cm}^{-1}, A_{1}=0.93$; $\omega_{2}=35187 \mathrm{~cm}^{-1}, \gamma_{2}=5612 \mathrm{~cm}^{-1}, A_{2}=0.61$. Thus, the sample is transparent in the visible and near-UV spectral range. We interpret these two bands as the two different electronic transitions activated when the excitation light in parallel to the nanotube axis, with the electric field vector $\boldsymbol{E}$ normal to the same axis. Note the very strong anisotropy in the absorption spectra recorded for the probe beam directed normal and parallel to the nanotube axis, to be discussed later.

\subsubsection{Emission spectra}

The emission spectra were studied for both the $\mathrm{T} 1$ and $\mathrm{T} 2$ samples, using the right-angles configuration, namely, the excitation was performed through the $0.2 \times 1.0 \mathrm{~cm}^{2}$ faces, and the emission recorded from the $0.02 \mathrm{~cm} \times 1.0 \mathrm{~cm}$ and $0.02 \mathrm{~cm} \times 0.2 \mathrm{~cm}$ faces for the $\mathrm{T} 1 \mathrm{sample}$, and from the $0.2 \mathrm{~cm} \times 1.0 \mathrm{~cm}$ and $0.2 \mathrm{~cm} \times 0.2 \mathrm{~cm}$ faces for the T2 sample. The excitation was performed at 280 and $357 \mathrm{~nm}$ into the broad absorption bands (see Fig. 4). Figs. 7 and 8 show the emission spectra for the respective samples $\mathrm{T} 1$ and $\mathrm{T} 2$.

We see in Figs. 7 and 8 that the emission spectrum recorded normal to the nanotube axis is quite broad, exhibiting a series of discrete bands, charts $(a, 1)$ and $(b, 1)$, while the spectrum recorded in the direction of the nanotube axis has a single narrow radiation band, charts $(a, 2)$ and $(b, 2)$. The respective band maxima, intensities and widths are listed in Table 2.

\subsubsection{The superemission}

Superemission may be observed if we create a population inversion between an excited state and a lower-lying (excited) state, when optical transitions between these two states are allowed. A higher excited state may be populated directly by optical absorption, or indirectly via relaxation from the initially created excited state to an intermediate state, lying above an unpopulated state. Provided the linear size of the active medium in one direction $(z)$ is much larger than that in the other two 

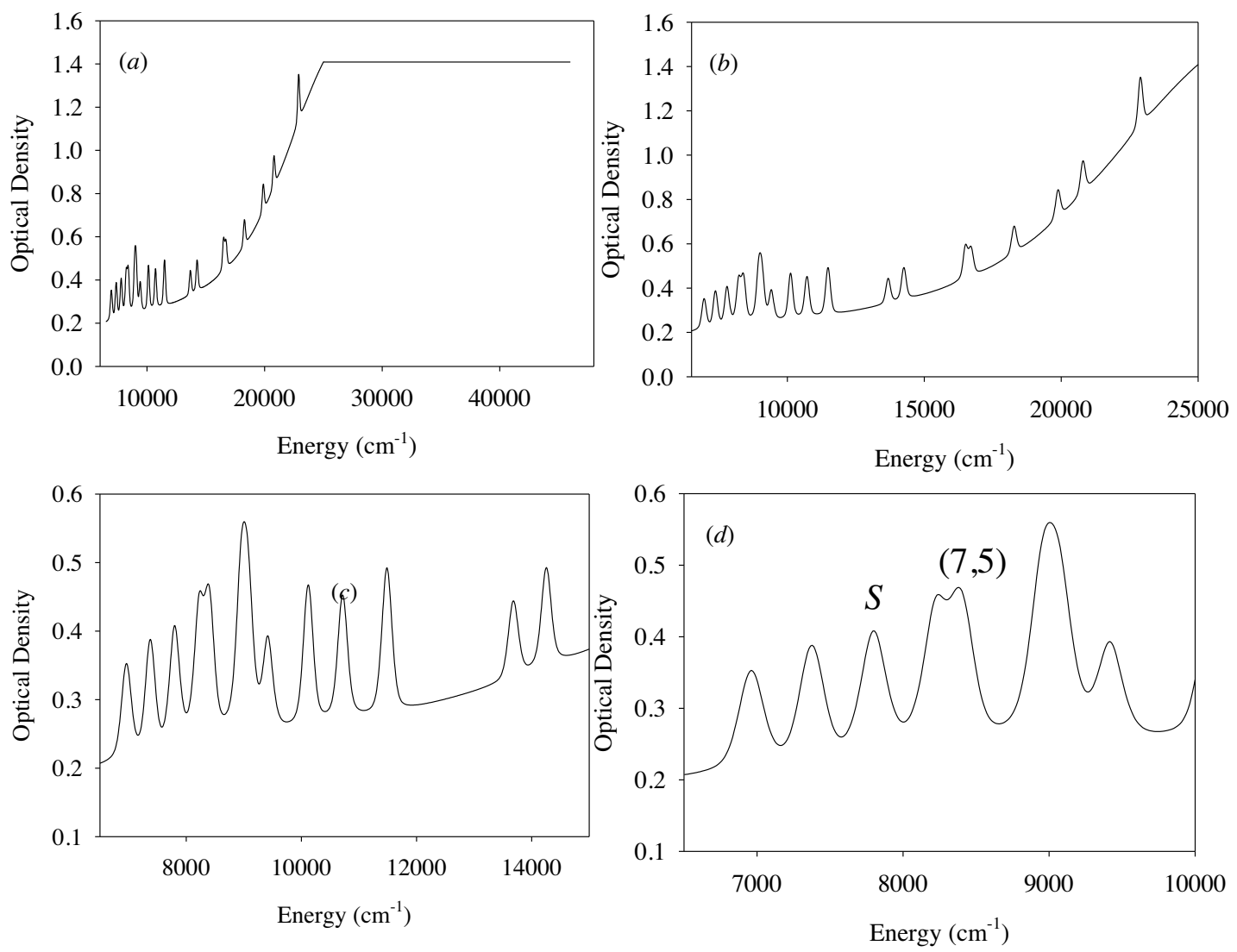

Fig. 5. The absorption spectra of the T2 sample, presented in different spectral ranges. The spectra were obtained with the probe beam normal to the SWCNT axis; the spectrum was recorded through the $0.2 \mathrm{~cm} \times 1.0 \mathrm{~cm}$ sample faces with the $0.2 \mathrm{~cm}$ optical thickness.

directions $(x, y)$, superemission will be observed in both the $-z$ and $+z$ directions.

All of the measurements of the superemission were carried out for the T1 sample, with the excitation beam passing through the $0.2 \mathrm{~cm} \times 1.0 \mathrm{~cm}$ faces, and the emis-

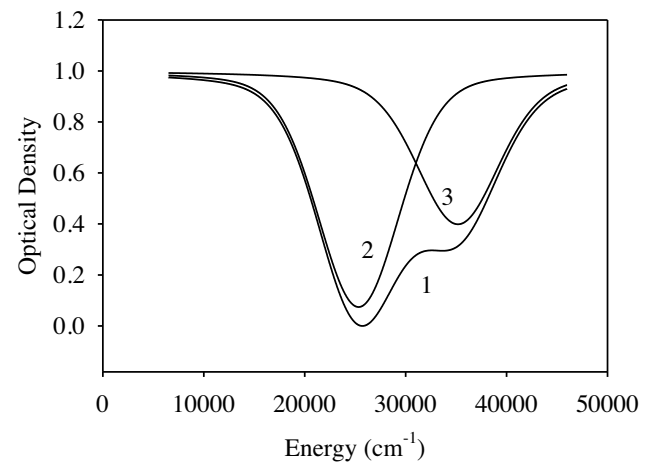

Fig. 6. The absorption spectrum of the T2 sample: 1, the experimental spectrum; 2, 3, the transmission band decomposed into two Gaussian subbands. The spectrum was recorded with the probe beam parallel to the SWCNT axis, through the $0.2 \mathrm{~cm} \times 0.2 \mathrm{~cm}$ sample faces with $1.0 \mathrm{~cm}$ optical thickness. sion recorded at the $0.02 \mathrm{~cm} \times 0.2 \mathrm{~cm}$ face. Thus, the excitation was performed normal to the nanotube axis, and the emission recorded in the direction of the axis. We recorded the laser-induced fluorescence excitation spectrum in the 270-290 nm range, using the frequencydoubled dye laser at $3.1 \mathrm{~mJ} /$ pulse, with the spectrum shown in Fig. 9.

Fig. 9 shows the laser induced fluorescence (LIF) spectrum recorded for the T1 sample excited normal to the VA SWCNT axis. The total emission, including light emitted in the near-IR, and UV-vis spectral ranges, was detected in the direction along the VA SWCNT axis. The spectrum was fitted by a superposition of Gaussian and Lorentzian functions:

$I_{L I F}=A_{G} e^{-\left(\omega-\omega_{0} / \sigma_{G}\right)^{2}}+\frac{A_{L}}{1+\left(\frac{\omega-\omega_{0}}{\sigma_{L}}\right)^{2}}$

where $A_{G}=73 \pm 8 ; \omega_{0}=35781 \pm 101 \mathrm{~cm}^{-1} ; \sigma_{G}=\sigma_{L}=$ $3302 \pm 217 \mathrm{~cm}^{-1} ; A_{G}=61 \pm 7$. Zero-slope straight-line background was used in the fitting procedure. Thus, this band coincides with the band 1 of the absorption 
Table 1

The maxima of the absorption bands and the band intensities. The SWCNT density of $1.72 \times 10^{13} \mathrm{~cm}^{-3}$ used in the cross-section calculations was estimated using the specification density value of $1.72 \times 10^{5} \mu^{-2}$ and the nanotube length of $1 \mathrm{~cm}$.

\begin{tabular}{|c|c|c|c|c|c|c|c|}
\hline \multirow{2}{*}{$\begin{array}{l}\text { Band } \\
\text { number }\end{array}$} & \multicolumn{3}{|c|}{ T1 sample $(l=0.02 \mathrm{~cm})$} & \multicolumn{3}{|c|}{$\mathrm{T} 2$ sample $(l=0.2 \mathrm{~cm})$} & \multirow{2}{*}{$\begin{array}{l}\text { Cross-section } \\
\text { ratio }\end{array}$} \\
\hline & $\begin{array}{l}\text { Band maximum, } \\
\mathrm{cm}^{-1}\end{array}$ & O.D. $\max$ & $\sigma, 10^{-11} \mathrm{~cm}^{2}$ & $\begin{array}{l}\text { Band maximum, } \\
\mathrm{cm}^{-1}\end{array}$ & O.D. max & $\sigma, 10^{-11} \mathrm{~cm}^{2}$ & \\
\hline 1 & 35,785 & 0.031 & $0.90 \pm 0.15$ & - & - & - & - \\
\hline 2 & 27,987 & 0.023 & $0.67 \pm 0.12$ & - & - & - & - \\
\hline 3 & 22,896 & 0.003 & $0.087 \pm 0.013$ & 22,896 & 0.304 & $0.88 \pm 0.16$ & $10.1 \pm 3.1$ \\
\hline 4 & 20,795 & 0.031 & $0.90 \pm 0.17$ & 20,795 & 0.200 & $0.58 \pm 0.10$ & $0.65 \pm 0.20$ \\
\hline 5 & 19,883 & 0.028 & $0.81 \pm 0.14$ & 19,883 & 0.191 & $0.56 \pm 0.10$ & $0.68 \pm 0.12$ \\
\hline 6 & 18,279 & 0.029 & $0.84 \pm 0.14$ & 18,279 & 0.209 & $0.61 \pm 0.11$ & $0.72 \pm 0.12$ \\
\hline 7 & 16,707 & 0.020 & $0.58 \pm 0.11$ & 16,707 & 0.113 & $0.33 \pm 0.08$ & $0.57 \pm 0.11$ \\
\hline 8 & 16,504 & 0.019 & $0.55 \pm 0.11$ & 16,504 & 0.139 & $0.40 \pm 0.09$ & $0.73 \pm 0.13$ \\
\hline 9 & 14,255 & 0.028 & $0.81 \pm 0.14$ & 14,255 & 0.217 & $0.63 \pm 0.10$ & $0.78 \pm 0.13$ \\
\hline 10 & 13,682 & 0.031 & $0.90 \pm 0.18$ & 13,682 & 0.200 & $0.58 \pm 0.10$ & $0.65 \pm 0.13$ \\
\hline 11 & 11,486 & 0.029 & $0.84 \pm 0.17$ & 11,486 & 0.391 & $1.14 \pm 0.21$ & $1.35 \pm 0.24$ \\
\hline 12 & 10,717 & 0.031 & $0.90 \pm 0.18$ & 10,717 & 0.383 & $1.11 \pm 0.23$ & $1.24 \pm 0.26$ \\
\hline 13 & 10,119 & 0.024 & $0.70 \pm 0.12$ & 10,119 & 0.391 & $1.14 \pm 0.24$ & $1.63 \pm 0.30$ \\
\hline 14 & 9418 & 0.021 & $0.61 \pm 0.11$ & 9418 & 0.191 & $0.56 \pm 0.11$ & $0.91 \pm 0.21$ \\
\hline 15 & 8953 & 0.051 & $1.48 \pm 0.24$ & 8953 & 0.461 & $1.34 \pm 0.25$ & $0.90 \pm 0.20$ \\
\hline 16 & 8400 & 0.019 & $0.55 \pm 0.11$ & 8400 & 0.226 & $0.66 \pm 0.11$ & $1.19 \pm 0.28$ \\
\hline 17 & 8222 & 0.028 & $0.81 \pm 0.15$ & 8222 & 0.270 & $0.78 \pm 0.13$ & $0.96 \pm 0.18$ \\
\hline 18 & 7798 & 0.063 & $1.83 \pm 0.27$ & 7798 & 0.235 & $0.68 \pm 0.12$ & $0.37 \pm 0.09$ \\
\hline 19 & 7375 & 0.035 & $1.02 \pm 0.20$ & 7375 & 0.217 & $0.63 \pm 0.11$ & $0.62 \pm 0.11$ \\
\hline 20 & 6960 & 0.035 & $1.02 \pm 0.23$ & 6960 & 0.200 & $0.58 \pm 0.09$ & $0.57 \pm 0.10$ \\
\hline
\end{tabular}

spectrum of this sample, with the superemission induced by the transitions from the states located close to $35781 \mathrm{~cm}^{-1}$, corresponding to the $0-0$ transition of this band located at ca. $32479 \mathrm{~cm}^{-1}$. This band is quite wide, and will be attributed to a specific excited state (exciton), as we shall discuss below.

The superemission spectra shown in Fig. 10 were recorded with the laser excitation at $35781 \mathrm{~cm}^{-1}$. Fig. 10 shows the superemission spectrum of the T1 sample excited by pulsed laser radiation at $35781 \mathrm{~cm}^{-1}$ normal to the VA SWCNT axis. The emission spectrum was analysed by passing the total emission directed along the VA SWCNT axis through the monochromator.

Fig. 10 shows that the bands at 7206 and $5257 \mathrm{~cm}^{-1}$ are observable with $35,781 \mathrm{~cm}^{-1}$ excitation, with the second band much weaker than the first.
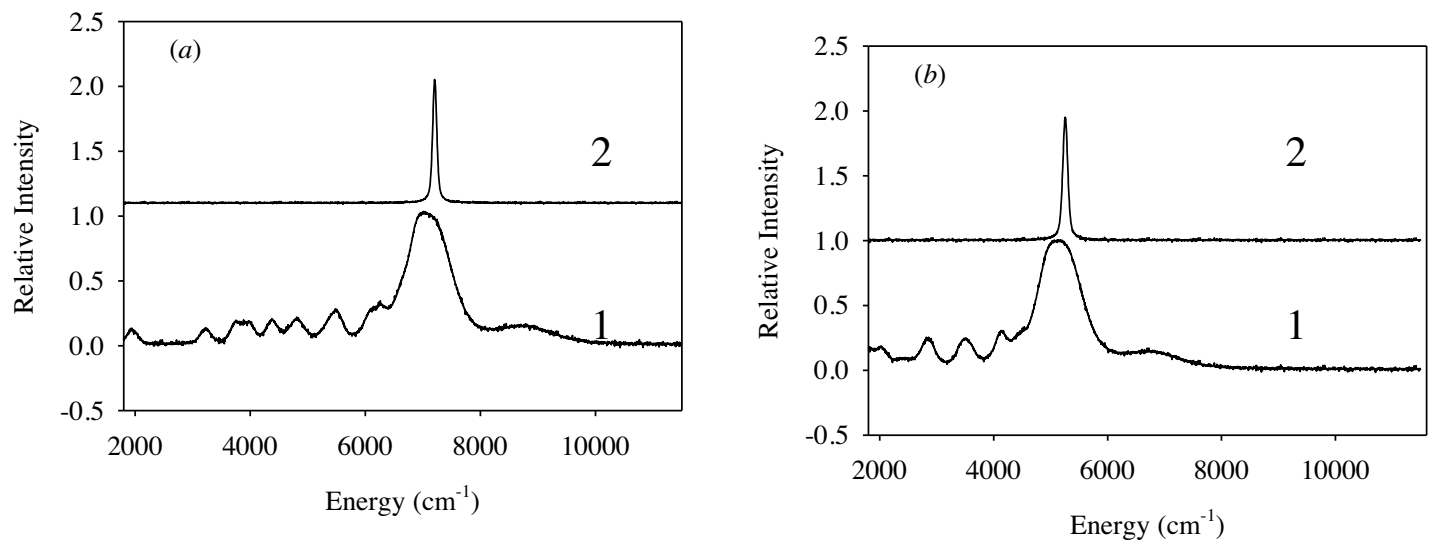

Fig. 7. The emission spectra of the T1 sample, excited through the $0.2 \mathrm{~cm} \times 1.0 \mathrm{~cm}$ faces: (a1), (a2), excited at $280 \mathrm{~nm}$; (b1), (b2), excited at $357 \mathrm{~nm}$; (a1), (b1), emission observed from the $0.02 \mathrm{~cm} \times 1.0 \mathrm{~cm}$ face; (a2), (b2), emission observed from the $0.02 \mathrm{~cm} \times 0.2 \mathrm{~cm}$ face. 

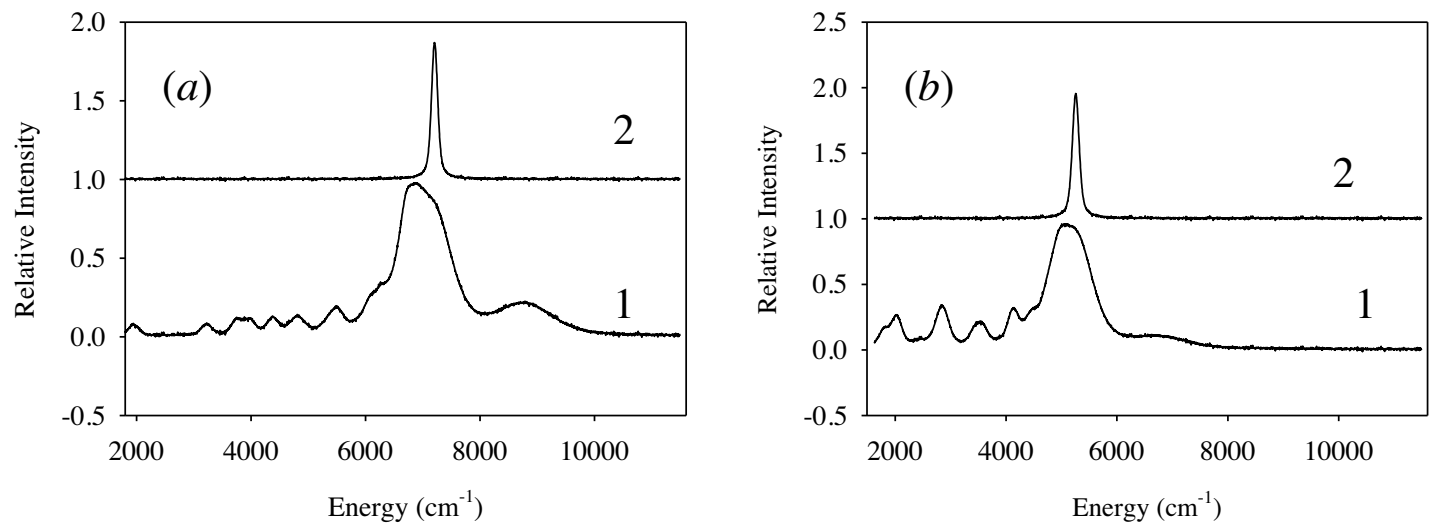

Fig. 8. Emission spectra of the T2 sample, excited through the $0.2 \mathrm{~cm} \times 1.0 \mathrm{~cm}$ faces: $(a 1),(a 2)$, excited at $280 \mathrm{~nm} ;(b 1),(b 2)$, excited at $357 \mathrm{~nm}$; $(a 1),(b 1)$, emission observed from the $0.2 \mathrm{~cm} \times 1.0 \mathrm{~cm}$ face; $(a 2),(b 2)$, emission observed from the $0.2 \mathrm{~cm} \times 0.2 \mathrm{~cm}$ face.

The SE kinetics was recorded for both bands using the $36,500 \mathrm{~cm}^{-1}$ excitation, with the waveforms shown in Fig. 11.

The first waveform was fitted by an exponential function:

$I_{S E}^{(1)}=A_{S E}^{(1)} e^{-t / \tau_{S E}^{(1)}}$

with $\tau_{S E}^{(1)}=152 \pm 21 \mathrm{~ns}$, while the second waveform-by a sum of two exponentials:

$I_{S E}^{(2)}=A_{S E}^{(2)}\left(e^{-\frac{t}{\tau_{S E}^{(2,1)}}}-e^{-\frac{t}{\tau_{S E}^{(2,2)}}}\right)$ with $\tau_{S E}{ }^{(2,1)}=209 \pm 36 \mathrm{~ns}$ and $\tau_{S E}^{(2,2)}=7.9 \pm 6.3 \mathrm{~ns}$, respectively. The fast rise time $\tau_{S E}{ }^{(2,2)}=7.9 \pm 6.3 \mathrm{~ns}$ describes the relaxation from the relaxed intermediate excited states populated by the intrasystem relaxation of the state excited by the $35781 \mathrm{~cm}^{-1}$ radiation. The two decay times $\tau_{S E}{ }^{(1)}=152 \pm 21$ and $\tau_{S E}{ }^{(2,1)}=209 \pm 36 \mathrm{~ns}$ correspond to the SE lifetime of the respective emitting state population and the decay of this state.

The superemission energy was measured for the $7206 \mathrm{~cm}^{-1}$ band pumped by the laser radiation at $35781 \mathrm{~cm}^{-1}(280 \mathrm{~nm})$. At the pumping energy of

Table 2

The emission band maxima, intensities and widths (see the spectra in Figs. 7 and8).

\begin{tabular}{|c|c|c|c|c|c|c|c|c|c|c|c|}
\hline \multicolumn{3}{|l|}{$a$} & \multicolumn{3}{|l|}{$b$} & \multicolumn{3}{|l|}{$c$} & \multicolumn{3}{|l|}{$d$} \\
\hline$\omega, \mathrm{cm}^{-1}$ & $A$ & $W, \mathrm{~cm}^{-1}$ & $\omega, \mathrm{cm}^{-1}$ & $A$ & $W, \mathrm{~cm}^{-1}$ & $\omega, \mathrm{cm}^{-1}$ & $A$ & $W, \mathrm{~cm}^{-1}$ & $\omega, \mathrm{cm}^{-1}$ & $A$ & $W, \mathrm{~cm}^{-1}$ \\
\hline \multicolumn{12}{|l|}{ Fig. 7} \\
\hline 1949 & 2 & 147 & \multirow[t]{11}{*}{7206} & \multirow[t]{10}{*}{139} & \multirow[t]{10}{*}{46} & 1798 & 2.3 & 146 & \multirow[t]{11}{*}{5257} & \multirow[t]{11}{*}{135} & \multirow[t]{11}{*}{55} \\
\hline 3222 & 2 & 153 & & & & 2026 & 1.8 & 155 & & & \\
\hline 3975 & 3.5 & 159 & & & & 2427 & 0.3 & 145 & & & \\
\hline 4376 & 3 & 135 & & & & 2871 & 5 & 160 & & & \\
\hline 4820 & 3 & 155 & & & & 3576 & 5 & 152 & & & \\
\hline 5525 & 4 & 155 & & & & 4317 & 2.7 & 177 & & & \\
\hline 6591 & 2 & 179 & & & & 4642 & 0.5 & 151 & & & \\
\hline 6996 & 7.9 & 617 & & & & 5257 & 18.6 & 572 & & & \\
\hline 7206 & 11.3 & 547 & & & & 6997 & 0.8 & 589 & & & \\
\hline 8945 & 1.4 & 593 & & & & & & & & & \\
\hline \multicolumn{8}{|l|}{ Fig. 8} & & & & \\
\hline 1948 & 2.4 & 141 & \multirow[t]{10}{*}{7206} & \multirow[t]{10}{*}{161} & \multirow[t]{10}{*}{59} & 1799 & 3.3 & 151 & \multirow[t]{10}{*}{5257} & \multirow[t]{10}{*}{139} & \multirow[t]{10}{*}{54} \\
\hline 3221 & 2.5 & 155 & & & & 2025 & 5.1 & 158 & & & \\
\hline 3975 & 3.1 & 154 & & & & 2425 & 0.5 & 149 & & & \\
\hline 4375 & 2.8 & 143 & & & & 2871 & 10 & 153 & & & \\
\hline 4820 & 2.7 & 157 & & & & 3575 & 5 & 152 & & & \\
\hline 5526 & 2.9 & 157 & & & & 4319 & 5 & 182 & & & \\
\hline 6590 & 2.3 & 175 & & & & 4640 & 0.7 & 153 & & & \\
\hline 6995 & 6.5 & 603 & & & & 5255 & 24.8 & 569 & & & \\
\hline 7205 & 12.1 & 560 & & & & 6997 & 1.1 & 599 & & & \\
\hline 8945 & 1.1 & 592 & & & & & & & & & \\
\hline
\end{tabular}




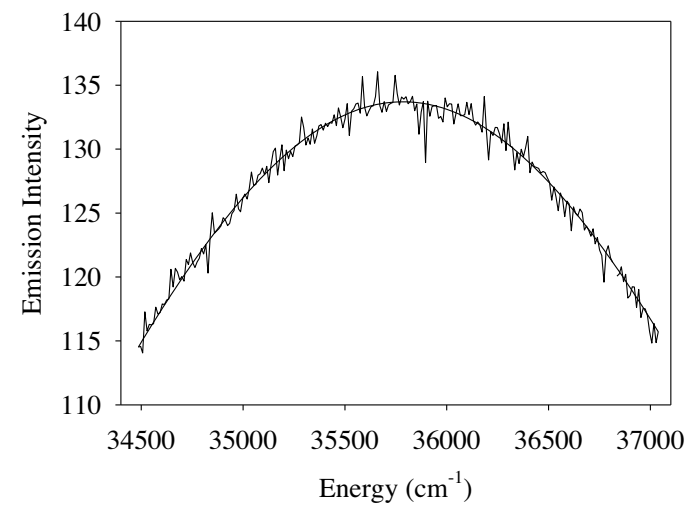

Fig. 9. Laser-induced fluorescence excitation spectrum of the T1 sample; the excitation beam is normal to the nanotube axis, with the emission observed along the axis.

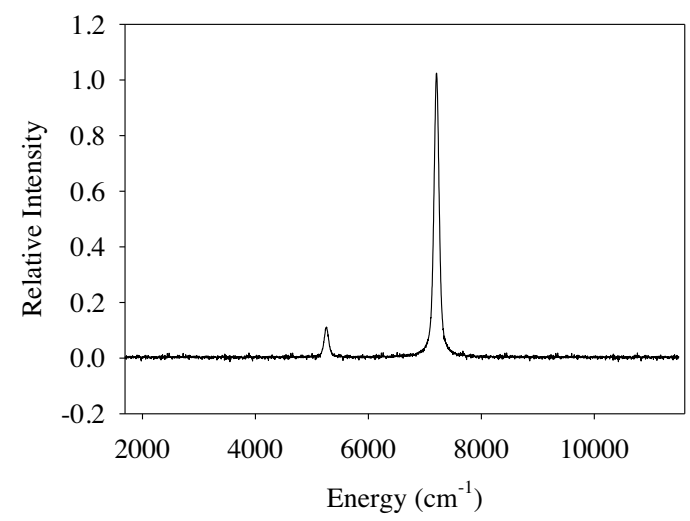

Fig. 10. The superemission spectra of the T1 sample recorded with laser excitation at $35,781 \mathrm{~cm}^{-1}$, where laser beam was directed perpendicular to the VA SWCNT axis. Emission was observed along the VA SWCNT axis.

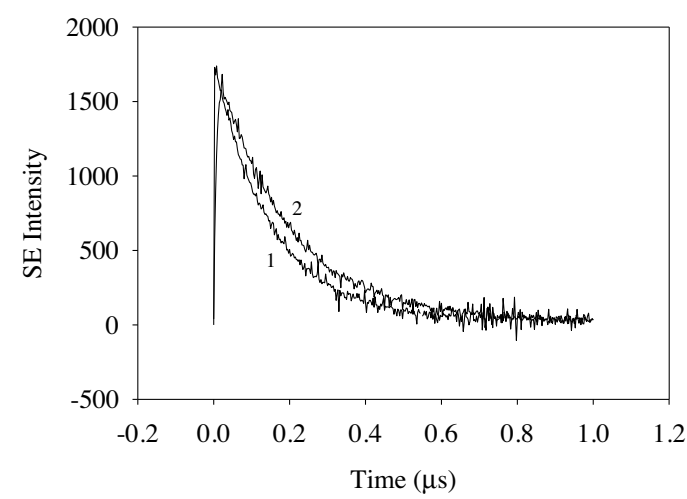

Fig. 11. The time evolution of the SE signal for the T1 sample, excited perpendicular to the VA SWCNT axis at $35,781 \mathrm{~cm}^{-1}$ and observed along the VA SWCNTs axis: (1) $7206 \mathrm{~cm}^{-1}$ and (2) $5257 \mathrm{~cm}^{-1}$ emission bands.
$3 \mathrm{~mJ} /$ pulse, the SE energy measured by the detector was $0.74 \mathrm{~mJ} /$ pulse, corresponding to the total SE energy of $1.48 \mathrm{~mJ} /$ pulse, with the energy density of $18.5 \mathrm{~mJ} \mathrm{~cm}^{-2} /$ pulse and the $\mathrm{SE}$ power density of $1.2 \times 10^{5} \mathrm{~W} \mathrm{~cm}^{-2} /$ pulse. These estimates show that the presently studied bundles of vertically aligned SWCNTs may be used as an active medium for lasers and nanolasers.

\section{The detailed analysis of the experimental data}

\subsection{The absorption spectrum}

We shall now analyze the spectrum shown in Fig. 4a. We shall assume that the vertically aligned SWCNTs have Gaussian distributions as regards their diameter $(d)$ and chirality angle $(\theta)$ :

$$
\begin{aligned}
& n(d)=n_{0}\left(d_{0}\right) e^{-\left(\frac{d-d_{0}}{\delta d}\right)^{2}} \\
& n(\theta)=n_{0}\left(\theta_{0}\right) e^{-\left(\theta-\theta_{0} / \delta \theta\right)^{2}}
\end{aligned}
$$

where $n_{0}$ is the density of the SWCNTs $\left(\mu \mathrm{m}^{-2}\right)$, $d_{0}=3.7 \mathrm{~nm}, \delta d$ the width of the Gaussian distribution, assumed equal to $1.3 \mathrm{~nm} ; n_{0}\left(\theta_{0}\right)$ a fitting parameter describing the maximum number density of SWCNTs with the $\theta_{0}$, chirality angle, $\delta \theta$ the width of the distribution, also a fitting parameter. To simplify the calculations, we assumed that the SWCNT bundle contains nanotubes with their diameter in the range $d_{0}-\delta d \leq d \leq d_{0}+\delta d$, and their chirality angles in the range $\theta_{0}-\delta \theta \leq \theta \leq \theta_{0}+\delta \theta$.

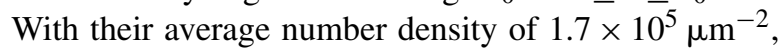
the SWCNTs are very densely packed. As we assumed that the SWCNT diameters all fall within the [2.4; 5.0] $\mathrm{nm}$ interval, we included the exchange interactions between the closest-neighbor nanotubes into the system Hamiltonian, with the elementary cell shown in Fig. 12.

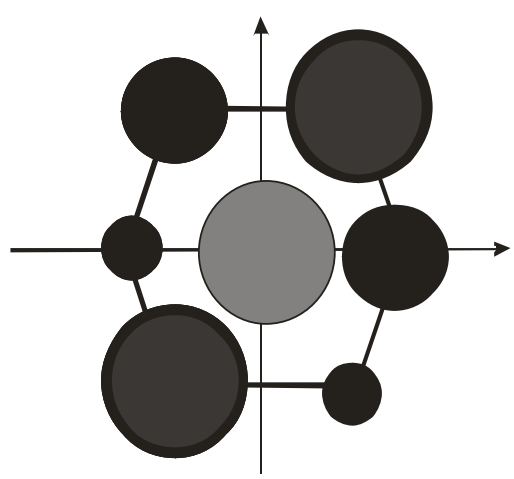

Fig. 12. The elementary cell in the nanotube bundle. 
Note that if the SWCNTs are randomly oriented, the exchange interaction between the closest neighbours may be neglected, whereas for well-organized VA SWCNTs these interactions play an important role, as the closet-neighbour nanotubes in the bundle are in contact all over their entire length, providing a large contact surface.

To calculate the exchange interaction between the closest neighbours (Fig. 12), we took into account such interactions between their respective carbon atoms. The carbon atoms involved in the exchange interactions between the two nanotubes are located close to the plane containing the axes of these nanotubes. The distance between these carbon atoms was calculated taking into account the structure of the elementary cell (Fig. 12) and the shortest distance between the surfaces of the closest neighbors. Analyzing the state structure of the elementary cell of Fig. 12, the distribution obtained by multiplication of the relationships (8) and (9)

$n(d, \theta)=n_{0}\left(d_{0}, \theta_{0}\right) e^{-\left(d-d_{0} / \delta d\right)^{2}} e^{-\left(\theta-\theta_{0} / \delta \theta\right)^{2}}$

was used, and the electronic and optical properties of the central nanotube calculated, accounting for the exchange interactions with its closest neighbors. Here, $n_{0}\left(d_{0}\right.$, $\left.\theta_{0}\right)=n_{0}\left(d_{0}\right) n_{0}\left(\theta_{0}\right)$. The results obtained for a selected nanotube with given values of $d$ and $\theta$ (directly dependent on the chirality parameters $m$ and $n$ ) were averaged statistically to obtain the optical absorption spectrum of an ensemble of vertically aligned SWCNTS. Denoting the absorption spectrum of a nanotube with the chirality parameters $(m, n)$ as $D_{m, n}(E)$, and using $n_{0}\left(d_{0}, \theta_{0}\right)=1$, the total absorption spectrum is given by [12]:

$D(E)=\sum_{m, n} D_{m, n}(E) e^{-\left(d_{m, n}-d_{0} / \delta d\right)^{2}} e^{-\left(\theta_{m, n}-\theta_{0} / \delta \theta\right)^{2}}$

In the calculations, we considered separately the nanotubes with each of the $(m, n)$ vectors, the elementary cell as shown in Fig. 12 was built using a randomly selected set of seven nanotubes, and the exchange interactions between these nanotubes were used as a perturbation mixing the electronic states of the nanotubes within the selected elementary cell. Thus, we calculated the structure of the electronic states and the optical properties of the central nanotube (shown in gray in Fig. 12) in the elementary cell. Next, we shall provide a detailed description of the presently used calculation procedures.

\subsubsection{Calculation methods}

We used the Su-Schrieffer-Heeger (SSH) model [50-57] in our numerical calculations of the electronic and optical properties of an individual nanotube. All calculations were carried out for the SWCNTs $20 \mathrm{~nm}$ long, as longer nanotubes required more calculation time. In the frameworks of the self-consistent field approximation, the model Hamiltonian may be written as follows:

$$
\begin{aligned}
H_{H F}= & \sum_{(i, j), s}\left(-t-\alpha \cdot y_{i j}\right) c_{i s}^{+} c_{i s}+\frac{K}{2} \sum_{i, j} y_{i j}^{2} \\
& +U \sum\left(\sum \rho_{i s} c_{i s}^{+} c_{i s}-\rho_{i \uparrow} \rho_{i \downarrow}\right)
\end{aligned}
$$

where the first and the second term determine kinetic energy of electrons ( $t, \alpha$, and $K$ are empirical parameters defined below), the third term determines the potential energy ( $U$ is an empirical parameter defined below), $c_{i s}{ }^{+}$ and $c_{i s}$ are the state creation and state annihilation operators. The eigenvalues and eigenvectors are obtained as the solutions of the secular equation

$\xi_{k s} v_{k s}=\sum_{(i, j)}\left(-t-\alpha \cdot y_{i j}\right) \psi_{k s}(j)-U \rho_{i s} \psi_{k s}(i)$

where

$\rho_{i s}=\left\langle c_{i s}^{+} c_{i s}\right\rangle=\sum_{k}^{\prime} \psi_{k s}(i) \psi_{k s}(j)$

is the electron density, with the summation done over the populated levels only.

Eq. (13) was obtained from the general Schrödinger equation:

$H_{H F} \sum_{k, s, i} C_{k s}^{(i)} \psi_{k s}(i)=E \sum_{k, s, i} C_{k s}^{(i)} \psi_{k s}(i)$

where $C_{k s}{ }^{(i)}$ are constants and $E$ is the energy. Combining the latter parameters with those included into the Hamiltonian, we obtain the expression for the $\xi_{k s} v_{k s}$ products.Using

$\sum_{i j} y_{i j}=0$

we obtain the self-consistency relation for $y_{i j}$ :

$y_{i j}=\frac{2 \alpha}{K} \sum_{k, s} \psi_{k s}(i) \psi_{k s}(j)-\Delta y$

where

$\Delta y=\frac{2 \alpha}{K N} \sum_{i, j} \sum_{k, s} \psi_{k s}(i) \psi_{k s}(j)$

here $N$ is the number of $\pi$-bonds. We used the same parameter values as proposed in [12]: $\alpha=6.3 \mathrm{eV} / \mathrm{A}$, $K=49 \mathrm{eV} / \mathrm{A}^{2}, \quad t=2.7 \mathrm{eV}, \quad U=7.4 \mathrm{eV}$. The first step includes calculations of the state structure of SWCNTs with $20 \mathrm{~nm}$ length and different diameters, assuming open hollow tubes. The next step of the numerical 
analysis includes calculations of the optical absorption properties of a SWCNT coupled by the exchange interaction with its six closest-neighbor nanotubes (Fig. 12). One-electron exchange interaction was used between the closest carbon atoms, with its matrix elements given by:

$$
\begin{aligned}
& \left\langle\psi_{\pi, k}\left|V_{\text {exch }}\right| \psi_{\pi, m}\right\rangle \\
& =-\sum_{i, j} C_{i}^{(\pi, k)} \cdot C_{j}^{(\pi, m)}\left\langle p_{i}^{(\pi, k)}\left|\frac{q_{C}^{2}}{r_{e}}\right| p_{j}^{(\pi, m)}\right\rangle
\end{aligned}
$$

here $C_{i}^{(\pi, k)}, C_{j}^{(\pi, m)}$ are the coefficients in the molecular orbitals (MO) of the interacting states of the two neighboring nanotubes, $p_{i, z}{ }^{(\pi, k)}$ and $p_{j, z}{ }^{(\pi, m)}$ are the $p_{z}$ atomic orbitals of the carbon atoms included into these MOs, and $i$ and $j$ number the carbon atoms located close to the plane passing through the axes of the nanotubes $k$ and $m$. The third step calculated the optical absorption properties of the ensemble of SWCNTs, based on their individual optical properties already obtained in the second step. The numerical calculations were performed using a home-made FORTRAN code, with the absorption bandwidth calculated from the density distribution of the two states that participate in the respective electronic transition. The calculation results are shown in Fig. 13. For comparison, the experimental spectrum is also presented in Fig. 13. First, the algorithm determines the molecular orbitals of the ground state SWCNT, and next calculates the exchange interactions between the closest SWCNT neighbors.

The band energies, widths and assignments are listed in Table 3.

The data shown in Fig. 13 and Table 3 correlate with an acceptable accuracy to the data shown in Fig. 4b (this experimental spectrum is also shown in Fig. 13)

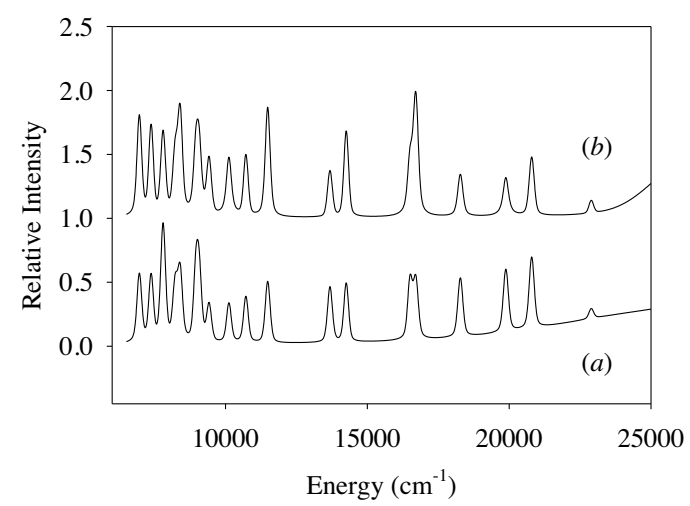

Fig. 13. The calculated absorption of a bundle of vertically aligned SWCNTs, with the probe beam normal to the SWCNT axis $(a)$. The experimental absorption spectra of the T1 sample (Fig. 4b) (b).

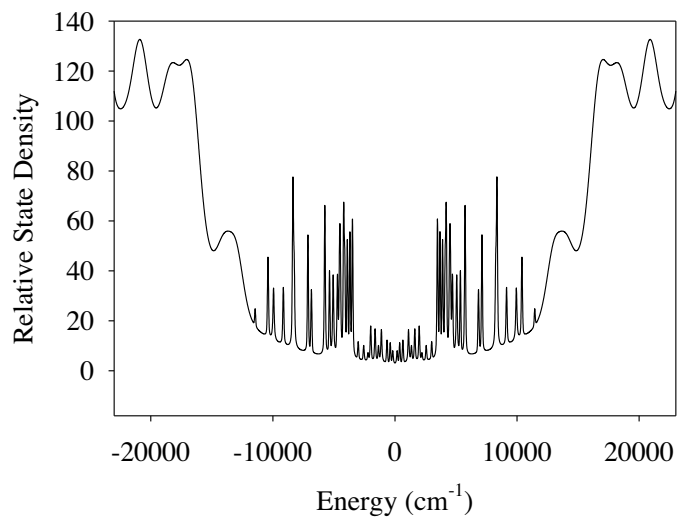

Fig. 14. Calculated electronic state density of a bundle of vertically aligned SWCNTs.

and Table 1. Therefore, we conclude that the presently developed theoretical approach may be used to describe the optical absorption properties of a bundle of vertically aligned SWCNTs. As we already noted, the band width was calculated according to the state density distribution in the system studied. Indeed, as may be inferred from the spectrum of Fig. 13 and the data of Table 3, these correlate quite well with the calculated electronic state density in the nanotube bundle, shown in Fig. 14.

In fact, comparing Fig. 14 with both Figs. 15 and 4a, we note a good correlation of the electronic state density with both calculated and experimental absorption spectra. Thus, ending this section, we conclude that

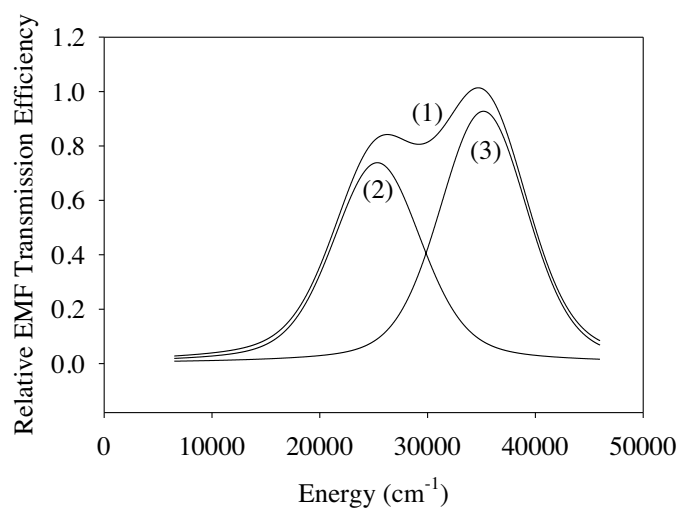

Fig. 15. The calculated light transmission spectrum by the vertically aligned SWCNT bundle; the resulting spectrum (1) was obtained by the superposition of two Gaussian bands with the following parameters: (2) $\omega_{1}=25,041 \mathrm{~cm}^{-1}, \gamma_{1}=5218 \mathrm{~cm}^{-1}, A_{1}=0.78$; and (3) $\omega_{2}=35,413 \mathrm{~cm}^{-1} ; \gamma_{2}=5358 \mathrm{~cm}^{-1} ; A_{2}=0.93$. For comparison, the experimental values are (see the spectrum in Fig. 5): $\omega_{1}=25,341 \mathrm{~cm}^{-1}$, $\gamma_{1}=5612 \mathrm{~cm}^{-1}, A_{1}=0.93 ;$ and $\omega_{2}=35,187 \mathrm{~cm}^{-1}, \gamma_{2}=5612 \mathrm{~cm}^{-1}$, $A_{2}=0.61$. 
Table 3

The band maxima, widths, and assignments for the calculated absorption spectrum of Fig. 14, and the SWCNT diameter used to obtain the respective band.

\begin{tabular}{|c|c|c|c|c|c|}
\hline Number & $\begin{array}{l}\text { Band maximum, } \\
\mathrm{cm}^{-1}\end{array}$ & Bandwidth, $\mathrm{cm}^{-1}$ & $\begin{array}{l}\text { Band assignment, } \\
(m, n)\end{array}$ & $\begin{array}{l}\text { Average nanotube } \\
\text { diameter }(\mathrm{nm})\end{array}$ & $\begin{array}{l}\text { Relative band } \\
\text { intensity }\end{array}$ \\
\hline 1 & 41,761 & 1611 & $(7,2)$ & 3.1 & 0.0886 \\
\hline 2 & 36,902 & 1721 & $(7,1)$ & 3.3 & 0.0541 \\
\hline 3 & 35,765 & 1625 & $(7,3)$ & 3.7 & 0.0497 \\
\hline 5 & 34,939 & 1603 & $(7,5)$ & 2.7 & 0.0515 \\
\hline 6 & 34,256 & 1731 & $(7,4)$ & 3.1 & 0.0637 \\
\hline 7 & 33,667 & 1613 & $(7,1)$ & 3.5 & 0.0606 \\
\hline 8 & 33,689 & 1651 & $(7,2)$ & 4.2 & 0.0591 \\
\hline 9 & 33,263 & 1636 & $(7,7)$ & 2.5 & 0.0583 \\
\hline 10 & 33,027 & 1710 & $(7,1)$ & 2.8 & 0.0587 \\
\hline 11 & 32,464 & 1627 & $(7,5)$ & 3.8 & 0.0547 \\
\hline 12 & 27,973 & 1612 & $(7,3)$ & 4.6 & 0.0330 \\
\hline 13 & 25,977 & 1612 & $(7,4)$ & 3.1 & 0.0237 \\
\hline 14 & 25,973 & 1612 & $(7,1)$ & 3.3 & 0.0212 \\
\hline 15 & 22,881 & 119 & $(5,5)$ & 3.9 & 0.0199 \\
\hline 16 & 20,785 & 129 & $(5,3)$ & 2.8 & 0.0191 \\
\hline 17 & 19,877 & 121 & $(4,1)$ & 2.6 & 0.0192 \\
\hline 18 & 18,279 & 122 & $(8,3)$ & 2.7 & 0.0217 \\
\hline 19 & 16,701 & 128 & $(6,5)$ & 4.6 & 0.0180 \\
\hline 20 & 16,721 & 127 & $(5,1)$ & 2.8 & 0.0205 \\
\hline 21 & 16,517 & 111 & $(9,5)$ & 3.0 & 0.0159 \\
\hline 22 & 14,243 & 173 & $(6,5)$ & 2.7 & 0.0131 \\
\hline 23 & 13,671 & 142 & $(10,5)$ & 2.7 & 0.0126 \\
\hline 24 & 11,479 & 121 & $(4,3)$ & 3.6 & 0.0230 \\
\hline 25 & 10,711 & 111 & $(8,1)$ & 2.8 & 0.0231 \\
\hline 26 & 10,103 & 143 & $(8,4)$ & 2.9 & 0.0212 \\
\hline 27 & 9407 & 129 & $(5,2)$ & 3.0 & 0.0180 \\
\hline 28 & 9057 & 129 & $(4,3)$ & 2.7 & 0.0362 \\
\hline 29 & 8389 & 153 & $(3,1)$ & 2.8 & 0.0215 \\
\hline 30 & 8209 & 128 & $(6,1)$ & 2.6 & 0.0205 \\
\hline 31 & 7779 & 127 & $(6,3)$ & 4.7 & 0.0181 \\
\hline 32 & 7363 & 111 & $(7,1)$ & 3.0 & 0.0141 \\
\hline 33 & 6947 & 157 & $(7,3)$ & 4.2 & 0.0197 \\
\hline
\end{tabular}

the presently developed approach may be used in the analysis of the optical absorption properties of the vertically-aligned SWCNT bundles, with the exchange interactions between the closest-neighbor nanotubes taken into account.

Note that the mathematical procedure used for the band assignment, with the results presented in Table 3, may produce non-unique results. That is, the same spectral band could be assigned to more than one set of the $m$ and $n$ values. However, we made test calculations, demonstrating that the results are indeed unique within the presently used diameter distribution of the nanotubes. A detailed numerical study was performed for the band $\# 30$ (8209 $\mathrm{cm}^{-1}, m=6, n=1$, and $\left.d=2.6 \mathrm{~nm}\right)$. Varying $m$ and $m$ in a broad range, we managed to obtain a band at $8213 \mathrm{~cm}^{-1}$ for $m=73, n=36$, and $d=7.9 \mathrm{~nm}$. The nanotube diameter distribution was cut off at $10 \mathrm{~nm}$ in these calculations. However, the intensity of this band should be close to zero, due to negligible concentration of such thick tubes in the sample. Thus, we conclude that the assignments of Table 3 are unique for the presently used diameter distribution of the SWCNTs.

\subsection{Analysis of the absorption spectrum of Fig. 6}

The absorption spectrum shown in Fig. 6 corresponds to an efficient light transmission by the vertically aligned SWCNTs in the $20,000-40,000 \mathrm{~cm}^{-1}$ spectral range, with the light transmitted in the direction parallel to the nanotube axis. To interpret these results, the earlier developed theory [53] was modified, taking into account the electronic state structure of the vertically aligned SWCNT bundle, and used to calculate the transmission spectrum of the SWCNT bundle. The presently reported theoretical approaches were used in the numerical calculations. The earlier developed FORTRAN code [57] 
was adapted to the present calculations, producing the calculated transmission spectrum shown in Fig. 15.

The calculated transmission spectrum was obtained by the superposition of two Gaussian bands (Fig. 15) that are in acceptable agreement with Gaussian bands describing the experimental absorption spectrum shown in Fig. 6. Thus, the presently developed theoretical approach is acceptable for the analysis of the transmission spectra of bundles of vertically aligned SWCNTs, using the light parallel to the nanotube axis.

To explain the presence of the two bands in the transmission spectrum, we should refer to the density of states distribution of Fig. 13, with the two strong peaks located in the $10,000-20,000 \mathrm{~cm}^{-1}$ energy range, both contributing to the emission propagating along the nanotube axis.

\subsubsection{The superemission divergence}

The SE beam divergence was measured using the method developed earlier [23]. All of the measurements were carried out for the T1 sample for the emission at $5257 \mathrm{~cm}^{-1}$. The measured beam divergence was $3.9 \times 10^{-3}$ and $5.2 \times 10^{-4} \mathrm{rad}$, along the 0.02 and $0.2 \mathrm{~cm}$ edges of the sample, respectively. The limiting beam divergence was estimated using

$\vartheta=\frac{\lambda}{\pi d}$

where $\lambda$ is the SE wavelength and $d$ is the geometrical size of the SE beam at the output face of the sample. The calculated values are $7.6 \times 10^{-4}$ and $7.6 \times 10^{-5} \mathrm{rad}$, with the experimental values exceeding the theoretic estimates by the factor of 3.8 and 6.8, respectively, as commonly occurs for the superemission. Thus, the maximum beam divergence was ca. $3.9 \times 10^{-3}$ rad for the T1 sample.

\section{Discussion}

We report that the vertically aligned SWCNTs have very interesting optical properties. We recorded the superemission of the nanotube bundle upon laser excitation. The superemission spectrum has narrow emission bands; we presume that the emission bandwidth may be reduced still further by producing nanotubes with more homogeneous properties. We found that the bundle of the VA SWCNTs, the T1 sample, has high superemission energy and power density in the direction parallel to the nanotube axis, with the emission exiting through the $0.02 \mathrm{~cm} \times 0.2 \mathrm{~cm}$ faces.

The superemission properties of the VA SWCNTs may be compared with those obtained for the VA singleand multi-wall carbon nanotubes [54-56]. Nojeh et al.
[54-56] found that such nanotubes may be used as light concentrators, i.e. directing the excitation light normal to the nanotube axis, high-density radiation exits at the ends of the nanotube. These effects are similar to what we observed earlier in metal nanolayers [18].

The results presently obtained for the vertically aligned SWCNTs are the first step to building a nanolaser. Lin et al. [57] investigated nanoscale optical devices for classical and quantum photonics. They discussed random lasers, where lasing relies on random multiple scattering. Presently we have a well-organized system, analyzed using the theoretical methods based on the SSH theory successfully applied by Prylutsky et al. [12] to the optical spectra of SWCNTs. We modified their approach, by including the exchange interaction between the closest-neighbor nanotubes. The methods developed describe the optical spectra of the vertically aligned SWCNTs with an acceptable accuracy. Note that calculated state density diagram has a specific structure, quite different from that reported by Prylutsky et al. [12]. Most probably, these differences result from the exchange interactions between the VA SWCNTs, closely packed in our samples.

As it was mentioned above, the emission spectra and the excited state dynamics of the isolated SWCNTs were extensively studied earlier [28-46]. Taking into account that the photoemission quantum yield for the transition between the quantum states $|i\rangle$ and $|j\rangle$ is given by:

$\varphi_{i j}(\lambda)=\frac{\left(\tau_{e m, i j}\right)^{-1}}{\left(\tau_{e m, i j}\right)^{-1}+k_{n r}(E)}$

where $\tau_{e m, i j}{ }^{-1}$ is the inverse radiative lifetime of the transition of interest and $k_{n r}(E)$ is the nonradiative rate constant describing the irreversible radiationless transitions from the state $|j\rangle$. It has been found earlier [31-35] for isolated SWCNTs that the relationship

$\left(\tau_{e m, i j}\right)^{-1}<<k_{n r}(E)$

is satisfied for the high-energy excited states, while for the low-energy excited states we have:

$\left(\tau_{e m, i j}\right)^{-1}<k_{n r}(E)$

The low-energy excited states are populated due to the relaxation of the high-energy excited states, typically induced by the exciton-phonon interaction. Presently we reported the emission spectra of the VA bundles of SWCNTs for the first time. The observed photoemission apparently results from the cooperative effects operating in the samples studied. These cooperative effects may be created by strong interactions between the SWCNTs organized into the VA bundle of macroscopic size. 
Therefore, we do not have the microscopic quantum system any more, expecting different properties. The excited state energy and dynamics in such macroscopic systems may be dramatically different as compared to those of isolated SWCNTs. Therefore, presently we are unable to exactly determine the nature of the optical transitions that are responsible for the observed superemission.

We address the reader to our previous publication [24] for the detailed discussion of the methods presently used to calculate the transmission spectra in the longitudinal direction.

\section{Conclusions}

In the present study, we investigated the NIR superemission in bundles of vertically aligned SWCNTs. We report that superemission is generated in the direction of the nanotube axis, when the pumping radiation is normal to it. At the $3 \mathrm{~mJ} /$ pulse of the pumping laser energy, we obtained the SE at $0.74 \mathrm{~mJ} /$ pulse in the direction of the detector, corresponding to the total of $1.48 \mathrm{~mJ} /$ pulse. The energy and the power density were, respectively, $18.5 \mathrm{~mJ} \mathrm{~cm}^{-2} /$ pulse and $1.2 \times 10^{5} \mathrm{~W} \mathrm{~cm}^{-2} /$ pulse. These results show that vertically aligned SWCNT bundles should be a good active medium for laser and nanolaser systems. The presently developed theoretical models were successfully used to fit both absorption and emission spectra of the nanotube bundles. The divergence of the superemission was quite low, thus we have effectively obtained a single-pass laser, with the nanotube bundle being an efficient radiation energy concentrator.

\section{Acknowledgements}

The authors are grateful for the PR NASA EPSCoR grant (NASA Cooperative Agreement NNX13AB22A) to V.M.

\section{References}

[1] X. Wang, Q. Li, J. Xie, Z. Jin, J. Wang, Y. Li, K. Jiang, S. Fan, Fabrication of Ultralong and Electrically Uniform Single-Walled Carbon Nanotubes on Clean Substrates, Nano Lett. 9 (2009) 3137-3141, http://dx.doi.org/10.1021/n1901260b.

[2] R. Martel, V. Derycke, C. Lavoie, J. Appenzeller, K. Chan, J. Tersoff, P. Avouris, Ambipolar electrical transport in semiconducting single-wall carbon nanotubes, Phys. Rev. Lett. 87 (2001) 256805, http://dx.doi.org/10.1103/PhysRevLett.87.256805.

[3] M.S. Dresselhaus, Nanotechnology: new tricks with nanotubes, Nature 391 (1998) 19-23.

[4] C.T. White, J.W. Mintmire, Density of states reflects diameter in nanotubes, Nature 394 (1998) 29-37.

[5] J.W.G. Wildoer, et al., Electronic structure of atomically resolved carbon nanotube, Nature 391 (1998) 59-67.
[6] R. Saito, M. Fujita, G. Dresselhaus, M.S. Dresselhaus, Tightbinding model for carbon nanotubes from ab initio calculations, Phys. Rev. B Condens. Matter 46 (1992) 1804-1921.

[7] C.L. Kane, E.J. Mele, Ratio problem in single carbon nanotube fluorescence spectroscopy, Phys. Rev. Lett. 90 (2003) 207401-207407.

[8] S. Yokojima, G.H. Chen, Linear-scaling localized-density-matrix method for the ground and excited states of one-dimensional molecular systems, Chem. Phys. Lett. 300 (1999) 540-549.

[9] W.Z. Liang, S. Yokojima, D.H. Zhou, G.H. Chen, Localizeddensity-matrix method and its application to carbon nanotubes, J. Phys. Chem. A 104 (2000) 2445-2453.

[10] W. Liang, G. Chen, Z. Li, Z.-K. Tang, Absorption spectra and chirality of single-walled $4 \AA$ carbon nanotubes, Appl. Phys. Lett. 80 (2000) 3415-3427.

[11] Y. Zhao, X.J. Wang, C.-C. Ma, G.H. Chen, Absorption spectral feature of single wall carbon nanotubes, Chem. Phys. Lett. 387 (2004) 149-154.

[12] Yu.I. Prylutsky, O.V. Ogloblya, P. Sharfe, Computer modelling of the optical absorption spectrum of single-walled carbon nanotube bundles, ISSN 0503-1265, Ukr. J. Phys. 49 (2004) A17-A28.

[13] M. Meyyappan, Carbon Nanotubes: Science and Applications, July 28, 2004 by CRC Press, Reference - 310 Pages - 188 B/W Illustrations ISBN 9780849321115 - CAT\# 2111.

[14] A.A. Tonkikh, V.I. Tsebro, E.A. Obraztsova, K. Suenaga, H. Kataura, A.G. Nasibulin, E.I. Kauppinen, E.D. Obraztsova, Metallization of single-wall carbon nanotube thin films induced by gas phase iodination, Carbon 94 (2015) 768-774, http://dx.doi.org/10.1016/j.carbon.2015.07.062.

[15] A. Roch, L. Stepien, T. Roch, I. Dani, C. Leyens, O. Jost, A. Leson, Optical absorption spectroscopy and properties of single walled carbon nanotubes at high temperature, Synth. Metals 197 (2014) 182-187.

[16] A.V. Naumov, S. Ghosh, D.A. Tsyboulski, S.M. Bachilo, R.B. Weisman, One-dimensional silver nanostructures on single-wall carbon nanotubes, ACS Nano J. 5 (2011) 1639-1648.

[17] W. Zhou, D. Nakamura, H. Liu, H. Kataura, S. Takeyama, Relative ordering between bright and dark excitons in singlewalled carbon nanotubes, Sci. Rep. 4 (6999) (2014) 1-6, http://dx.doi.org/10.1038/srep06999.

[18] I.V. Khmelinskii, V.I. Makarov, Superemission of Cr Nanolayers, Mater. Res. Bull. (2016) (in print March 29).

[19] R. Saito, G. Dresselhaus, M.S. Dresselhaus, Physical Properties of Carbon Nanotubes, Imperial College Press, London, 1998.

[20] M.S. Dresselhaus, G. Dresselhaus, Ph. Avouris (Eds.), Carbon Nanotubes: Synthesis, Structure, Properties, and Applications, Springer-Verlag, New York, 2001.

[21] A. Ugawa, A. Rinzler, D.B. Tanner, Far-infrared gaps in single-wall carbon nanotubes, Phys. Rev. B 60 (1999) R11305-R11308.

[22] M.E. Itkis, S. Niyogi, M.E. Meng, M.A. Harmon, H. Hu, R.C. Haddon, Spectroscopic study of the Fermi level electronic structure of single-walled carbon nanotubes, Nano Lett. 2 (2002) $155-159$.

[23] C.L. Kane, E.J. Mele, Size, shape, and low energy electronic structure of carbon nanotubes, Phys. Rev. Lett. 78 (1997) 1932-1935.

[24] Th. Pichler, M. Knupfer, M.S. Golden, J. Fink, A. Rinzler, R.E. Smalley, Localized and delocalized electronic states in singlewall carbon nanotubes, Phys. Rev. Lett. 80 (1998) 4729-4732.

[25] Y. Murakami, E. Einarsson, T. Edamura, S. Maruyama, Polarization dependence of the optical absorption of single-walled carbon nanotubes, Phys. Rev. Lett. 94 (2005), 087402/1-087402/4.1. 
[26] J.B. Birks, Photophysics of Aromatic Molecules, Wiley, London, 1970.

[27] Y.-Z. Ma, J. Stenger, J. Zimmerman, S.M. Bachilo, R.E. Smalley, R.B. Weisman, G.R. Fleming, Ultrafast carrier dynamics in single-walled carbon nanotubes probed by femtosecond spectroscopy, J. Chem. Phys. 120 (2004) 3368-3372.

[28] S. Reich, M. Dworzak, A. Hoffmann, C. Thomsen, M.S. Strano, Excited-state carrier lifetime in single-walled carbon nanotubes, Phys. Rev. B 71 (2005), 033402/1 - 033402/4. 25.

[29] J.-P. Yang, M.M. Kappes, H. Hippler, A.-N. Unterreiner, Femtosecond transient absorption spectroscopy of single-walled carbon nanotubes in aqueous surfactant suspensions: determination of the lifetime of the lowest excited state, Phys. Chem. Chem. Phys. 7 (2005) 512-517.

[30] G.N. Ostojic, S. Zaric, J. Kono, M.S. Strano, V.C. Moore, R.H. Hauge, R.E. Smalley, Interband recombination dynamics in resonantly excited single-walled carbon nanotubes, Phys. Rev. Lett. 92 (2004), 117402/1-117402/4.

[31] F. Wang, G. Dukovic, L.E. Brus, T.F. Heinz, The optical resonances in carbon nanotubes arise from excitons, Science 5723 (2005) 838-841.

[32] A.M. Rao, E. Richter, S. Bandow, B. Chase, P.C. Eklund, K.A. Williams, S. Fang, K.R. Subbaswamy, M. Menon, A. Thess, R.E. Smalley, G. Dresselhaus, M.S. Dresselhaus, Diameter-selective Raman scattering from vibrational modes in carbon nanotubes, Science 275 (1997) 187-191.

[33] M.S. Dresselhaus, G. Dresselhaus, A. Jorio, A.G. Souza Filho, R. Saito, Raman spectroscopy on isolated single wall carbon nanotubes, Carbon 40 (2002) 2043-2061.

[34] C.L. Kane, E.J. Mele, Ratio problem in single carbon nanotube fluorescence spectroscopy, Phys. Rev. Lett. 90 (2003) 207401-207407.

[35] M. O'Connell, S.M. Bachilo, C.B. Huffman, V. Moore, M.S. Strano, E. Haroz, K. Rialon, P.J. Boul, W.H. Noon, C. Kittrell, J. Ma, R.H. Hauge, R.B. Weisman, R.E. Smalley, Band-gap fluorescence from individual single-walled carbon nanotubes, Science 297 (2002) 593-596.

[36] H. Htoon, M.J. O'Connell, S.K. Doorn, V.I. Klimov, Single carbon nanotubes probed by photoluminescence excitation spectroscopy: the role of phonon-assisted transitions, Phys. Rev. Lett. 94 (2005), 127403/1-127403/4.

[37] M. Jones, C. Engtrakul, W.K. Metzger, R.J. Ellingson, A.J. Nozik, M.J. Heben, G. Rumbles, Analysis of photoluminescence from solubilized single-walled carbon nanotubes, Phys. Rev. B 71 (2005), 115426/1-115426/9.

[38] J. Lefebvre, P. Finnie, Y. Homma, Temperature-dependent photoluminescence from single-walled carbon nanotubes, Phys. Rev. B 70 (2004), 045419/1-045419/8.

[39] R.B. Weisman, S.M. Bachilo, D. Tsyboulski, Fluorescence spectroscopy of single-walled carbon nanotubes in aqueous suspension, Appl. Phys. A 78 (2004) 1111-1116.

[40] J. Lefebvre, J.M. Fraser, Y. Homma, P. Finnie, Photoluminescence from single-walled carbon nanotubes: a comparison between suspended and micelle-encapsulated nanotubes, Appl. Phys. A 78 (2004) 1107-1110.

[41] J. Lefebvre, J.M. Fraser, P. Finnie, Y. Homma, Photoluminescence from an individual single-walled carbon nanotube, Phys. Rev. B 69 (2004), 075403/1-075403/5.

[42] T. Ando, Excitons in carbon nanotubes, J. Phys. Soc. Jpn. 66 (1997) 1066-1073.
[43] C.D. Spataru, S. Ismail-Beigi, L.X. Benedict, S.G. Louie, Excitonic effects and optical spectra of single-walled carbon nanotubes, Phys. Rev. Lett. 92 (2004), 077402/1-077402/4.

[44] V. Perebeinos, J. Tersoff, P. Avouris, Scaling of excitons in carbon nanotubes, Phys. Rev. Lett. 92 (2004), 257402/1-257402/4.

[45] Y.-Z. Ma, L. Valkunas, S.L. Dexheimer, S.M. Bachilo, G.R. Fleming, Femtosecond spectroscopy of optical excitations in single-walled carbon nanotubes: evidence for exciton-exciton annihilation, Phys. Rev. Lett. 94 (2005), 157402/1-157402/4.

[46] V. Perebeinos, J. Tersoff, P. Avouris, Radiative lifetime of excitons in carbon nanotubes, Los Alamos National Laboratory Preprint Archive, Condens. Matter (2005), 0506775/1-0506775/4.

[47] C. Manzoni, A. Gambetta, E. Menna, M. Meneghetti, G. Lanzani, G. Cerullo, Intersubband exciton relaxation dynamics in single-walled carbon nanotubes, Phys. Rev. Lett. 94 (2005), 207401/1-207401/4.

[48] H. Liu, T. Tanaka, H. Kataura, Optical isomer separation of single-chirality carbon nanotubes using gel column chromatography, Nano Lett. 14 (2014) 6237-6243, http://dx.doi.org/10.1021/n15025613.

[49] H. Kuzmany, W. Plank, M. Hulman, Determination of SWCNT diameters from the Raman response of the radial breathing mode, Eur. Phys. J. B 22 (2001) P307-P320.

[50] Y.-S. Lim, A.R.T. Nugraha, S.-J. Cho, M.-Y. Noh, E.-J. Yoon, H. Liu, J.-H. Kim, H. Telg, E.H. Haroz, G.D. Sanders, S.H. Baik, H. Kataura, S.K. Doorn, C.J. Stanton, R. Saito, J. Kono, T. Joo, Ultrafast generation of fundamental and multiple-order phonon excitations in highly-enriched $(6,5)$ singlewall carbon nanotubes, Nano Lett. 14 (2014) 1426-1432, http://dx.doi.org/10.1021/n1404536b.

[51] M. Sauer, H. Shiozawa, P. Ayala, H. Ruiz-Soria, X. Liu, K. Yanagi, H. Kataura, S. Krause, T. Pichler, Environmental stability of ferrocene filled in purely metallic single-walled carbon nanotubes, Phys. Status Solidi B 250 (2013) 2599-2604, http://dx.doi.org/10.1002/pssb.201300124.

[52] T. Hertel, R. Fasel, G. Moos, Exciton transfer and propagation in carbon nanotubes studied by near-field optical microscopy, Appl. Phys. A. 75 (2002) P449-P465.

[53] V. Makarov, L. Zueva, T. Golubeva, E. Korneeva, I. Khmelinskii, M. Inyushin, Quantum mechanism of light transmission by the intermediate filaments in some specialized optically transparent cells, Neurophotonics J. (2016) (in press).

[54] M. Chang, P. Yaghoobi, W. Khalid, A. Nojeh, The synthesis of vertically aligned multi-walled carbon nanotube forests by thermal chemical vapor deposition, in: A. Mishra (Ed.), Carbon Nanotubes: Synthesis, Properties and Applications, Nova Science Publishers Inc., 2012.

[55] A.H. Khoshaman, A.T. Koch, M. Chang, H.D.E. Fan, M.V. Moghaddam, A. Nojeh, Nanostructured thermionics for conversion of light to electricity: simultaneous extraction of device parameters, IEEE Trans. Nanotechnol. 14 (2015) 624-632.

[56] M. Vahdani Moghaddam, P. Yaghoobi, G.A. Sawatzky, A. Nojeh, Photon-impenetrable, electron-permeable: the carbon nanotube forest as a medium for multi-photon thermal-photoemission, ACS Nano 9 (2015) 4064-4069.

[57] J. Liu, P.D. Garcia, S. Ek, N. Gregersen, T. Suhr, M. Schubert, J. Mørk, S. Stobbe, P. Lodahl, Plasmon-induced hot carrier science and technology, Nat. Nanotechnol. 9 (2014) 285-289, http://dx.doi.org/10.1038/nnano.2014.34. 